\title{
Papers
}

\section{Bleeding and pneumonia in intensive care patients given ranitidine and sucralfate for prevention of stress ulcer: meta-analysis of randomised controlled trials}

\author{
A Messori, S Trippoli, M Vaiani, M Gorini, A Corrado
}

\begin{abstract}
Objectives To determine the effectiveness of ranitidine and sucralfate in the prevention of stress ulcer in critical patients and to assess if these treatments affect the risk of nosocomial pneumonia.

Design Published studies retrieved through Medline and other databases. Five meta-analyses evaluated effectiveness in terms of bleeding rates (A: ranitidine $v$ placebo; B: sucralfate $v$ placebo) and infectious complications in terms of incidence of nosocomial pneumonia (C: ranitidine $v$ placebo; D: sucralfate $v$ placebo; E: ranitidine $v$ sucralfate). Trial quality was determined with an empirical ad hoc procedure.

Main outcome measures Rates of clinically important gastrointestinal bleeding and nosocomial pneumonia (compared between the two study arms and expressed with odds ratios specific for individual studies and meta-analytic summary odds ratios).

Results Meta-analysis A (five studies) comprised 398 patients; meta-analysis $\mathrm{C}$ (three studies) comprised 311 patients; meta-analysis D (two studies) comprised 226 patients: and meta-analysis E (eight studies) comprised 1825 patients. Meta-analysis B was not carried out as the literature search selected only one clinical trial. In meta-analysis A ranitidine was found to have the same effectiveness as placebo (odds ratio of bleeding $0.72,95 \%$ confidence interval 0.30 to 1.70 , $\mathrm{P}=0.46$ ). In placebo controlled studies (meta-analyses $\mathrm{C}$ and $\mathrm{D}$ ) ranitidine and sucralfate had no influence on the incidence of nosocomial pneumonia. In comparison with sucralfate, ranitidine significantly increased the incidence of nosocomial pneumonia (meta-analysis $\mathrm{E}: 1.35,1.07$ to $1.70, \mathrm{P}=0.012$ ). The mean quality score in the four analyses (on a 0 to 10 scale) ranged from 5.6 in meta-analysis $\mathrm{E}$ to 6.6 in meta-analysis A.

Conclusions Ranitidine is ineffective in the prevention of gastrointestinal bleeding in patients in intensive care and might increase the risk of pneumonia. Studies on sucralfate do not provide conclusive results. These findings are based on small numbers of patients, and firm conclusions cannot presently be proposed.
\end{abstract}

\begin{abstract}
Introduction
Ranitidine and sucralfate are widely used to prevent stress ulcers in patients admitted to intensive care units. ${ }^{1}$ A meta-analysis published by Cook et al in 1996 showed that $\mathrm{H}_{2}$ receptor antagonists (such as cimetidine and ranitidine together) are more effective than placebo for this clinical indication. ${ }^{2}$ With regard to sucralfate, this meta-analysis found a small but significant reduction in overt bleeding but no effect on clinically important events. The meta-analysis did not resolve the question of an increased risk of nosocomial pneumonia related to the use of $\mathrm{H}_{2}$ receptor antagonists.

Several arguments emphasise the need for up to date information on this issue. Firstly, ranitidine has become the main $\mathrm{H}_{2}$ receptor antagonist used for prophylaxis for stress ulcers, and cimetidine has generally been abandoned ${ }^{1}$; secondly, new findings have been published on effectiveness and complications of ranitidine; and, thirdly, a meta-analytic comparison of ranitidine versus placebo has never been carried out, and as the comparison of sucralfate and placebo made by Cook et al gave no proof of the effectiveness of this

Laboratorio SIFO di
Farmacoeconomia,
Centro
Informazione
Farmaci, Servizio
Farmaceutico,
Azienda
Ospedaliera
Careggi, 50134
Florence, Italy
A Messori
coordinator
S Trippoli
coordinator
M Vaiani
research fellow
Unita' di Terapia
Intensiva
Respiratoria,
Azienda
Ospedaliera Careggi
M Gorini
staff physician
A Corrado
head
Correspondence to:
A Messori
md3439@mclink.it
BMJ 2000;321:1-7

BMJ 2000;321:1-7
\end{abstract} drug, ranitidine and sucralfate might both be ineffective. Another problem is that the most recent randomised studies on this topic did not include a group with no prophylaxis and compared supposedly active treatments with one another. ${ }^{34}$

We conducted a literature search to identify randomised trials, and we carried out a meta-analysis to update the results of Cook's study with regard to effectiveness and infectious complications.

\section{Methods}

Searching

Our Medline search covered the period from 1966 to 20 June 2000 and was based on four key words (stress, pneumonia, ranitidine, sucralfate) and on the extraction of studies published in English (see appendix). Randomised studies were identified by using the key words "randomized controlled trial" or "random" according to a validated literature search. ${ }^{5}$

This search was supplemented by examining the Iowa-IDIS system (Iowa Drug Information, Iowa University, United States) from 1966 to December 
Table 1 Results of Medline search and selection of randomised controlled trials in meta-analyses. Figures are numbers of trials

\begin{tabular}{|c|c|c|c|c|c|}
\hline & \multicolumn{2}{|c|}{ Effectiveness of drug v placebo } & \multicolumn{3}{|c|}{ Rate of pneumonia } \\
\hline & $\begin{array}{c}\text { Ranitidine } \\
\text { (analysis A) }\end{array}$ & $\begin{array}{c}\text { Sucralfate } \\
\text { (analysis B) }\end{array}$ & $\begin{array}{l}\text { Ranitidine v placebo } \\
\text { (analysis C) }\end{array}$ & $\begin{array}{l}\text { Sucralfate v placebo } \\
\text { (analysis D) }\end{array}$ & $\begin{array}{l}\text { Ranitidine v sucralfate } \\
\text { (analysis E) }\end{array}$ \\
\hline Identified and screened studies & 51 & 46 & 59 & 35 & 11 \\
\hline Studies excluded & $45^{*}$ & $42^{*}$ & $56^{\star}$ & $33^{*}$ & $3^{*}$ \\
\hline Potentially relevant trials for inclusion & 6 & 4 & 3 & 2 & 8 \\
\hline Studies excluded & $1^{24} \dagger$ & $3^{16-18} \dagger$ & 0 & 0 & 0 \\
\hline Trials included & $5^{11-15}$ & $1^{11}$ & $3^{121315}$ & $2^{1718}$ & $8^{319-26}$ \\
\hline
\end{tabular}

*Studies were excluded because they did not compare treatment options considered by meta-analysis in question $(A=16 ; B=18, C=12 ; D=14 ; E=1)$ or because objective of trial was not prophylaxis of stress ulcer $(A=29 ; B=23, C=44 ; D=19 ; E=1)$ or because they duplicated study already included in meta-analysis question $(A=0 ; B=1, C=0 ; D=0 ; E=1)$.

†Studies excluded because end point of bleeding did not meet definition of "clinically important bleeding" according to Cook et al. ${ }^{2}$

1999 and Drugdex (CD Rom Drugdex, vol 104, Micromedex, Englewood, Colorado, United States).

\section{Selection}

On the basis of the material produced by our search, we carried out five meta-analyses that evaluated data on effectiveness in terms of rates of bleeding (meta-analysis A: ranitidine $v$ placebo; meta-analysis B: sucralfate $v$ placebo) and data of infectious complications in terms of incidence of nosocomial pneumonia (meta-analysis C: ranitidine $v$ placebo; meta-analysis D: sucralfate $v$ placebo; meta-analysis $\mathrm{E}$ : ranitidine $v$ sucralfate). We investigated the issue of effectiveness only in studies with a control group that received no prophylaxis, while the issue of infectious complications was also assessed from comparative trials of ranitidine versus sucralfate. Eligible studies were included in meta-analysis A or B if they met the following criteria: patients were admitted to an intensive care unit or were undergoing mechanical ventilation, or both; randomised design; assessment of gastrointestinal bleeding. We excluded studies in which gastrointestinal bleeding did not meet the definition of "clinically important bleeding" according to Cook et al. ${ }^{2}$ In metaanalyses $\mathrm{C}, \mathrm{D}$, and $\mathrm{E}$ the inclusion criterion gastrointestinal bleeding was replaced by the assessment of pneumonia. The definitions of pneumonia adopted by the individual investigators were recorded but were not assumed to be an inclusion or exclusion criterion.

\section{Data extraction}

Data were extracted by one reviewer (MV) with a structured form and checked for accuracy by a second reviewer (ST). Differences were resolved by consensus.

\section{Assessment of quality of trials}

We assessed methodological quality of the trials using a procedure similar to that adopted by Cook et al. ${ }^{2}$ Five items were evaluated for each trial (patient selection, patient characteristics, randomisation, blinding, definition of bleeding or of pneumonia). Methodological quality was graded for each of the five items on a scale of 0,1 , or 2 (maximum score $=10$ ). Three observers independently assessed quality (AM, MV, MG). Differences were resolved by consensus.

\section{Qualitative data synthesis}

Descriptive data for each trial included characteristics of participants, details of intervention, and definition of outcomes. Our assessment of clinical heterogeneity was focused in particular on a comparison of the definitions of bleeding and pneumonia across the trials.
Quantitative data synthesis

With regard to the two end points, the odds ratio was used as the principal measure for comparing the treatment effect within each trial. The results specific for trials were combined through standard meta-analytic techniques $^{6-8}$ to produce the summary odds ratio together with an assessment of the statistical intertrial heterogeneity. The meta-analysis calculations were based both on a fixed effect model ${ }^{6}{ }^{7}$ and on a random effect model. ${ }^{6}{ }^{8}$ The $95 \%$ confidence intervals for the odds ratio of individual studies were computed according to Wolf ${ }^{9}$ (or by the "rule of three" ${ }^{10}$ when zeros were present). To avoid the problems of bias and instability associated with estimation of odds ratios, 0.5 was added to each cell of the fourfold tables in keeping with Cook et al. $^{2}$ Heterogeneity was assessed as previously described. $^{7}$

\section{Results}

Table 1 shows the trial flow for the five meta-analyses. There were 398 patients in meta-analysis A (five studies), 54 in meta-analysis B (one study), 311 in meta-analysis C (three studies), 226 in meta-analysis D (two studies), and 1825 in meta-analysis E (eight studies). Table 2 shows descriptive data for all the trials included in our meta-analyses.

Effectiveness of ranitidine $v$ placebo (meta-analysis A)-Our literature search identified five trials, ${ }^{11-15}$ three of which had already been included by Cook et al. ${ }^{11-13}$ The mean (SD) quality score for these trials was 6.6 (0.9). With respect to the end point of clinically important bleeding, this meta-analysis (table 3) failed to show any significant benefit of ranitidine (summary odds ratio $0.72,95 \%$ confidence interval 0.30 to 1.70 , $\mathrm{P}=0.46$ for fixed effect model; $0.95,0.37$ to 2.43 , $\mathrm{P}=0.92$ for random effect model; $\chi^{2}$ for heterogeneity $6.8, \mathrm{df} 4, \mathrm{P}=0.15$ ).

Effectiveness of sucralfate $v$ placebo (meta-analysis $B$ - -Our literature search found the same randomised trials that had already been examined by Cook et al (three trials ${ }^{16-18}$ for the end point of overt bleeding and one trial ${ }^{11}$ for the end point of clinically important gastrointestinal bleeding). Because our analysis considered the end point of clinically important bleeding, only one trial ${ }^{11}$ met the inclusion criteria and so no meta-analysis was carried out. The quality score of the trial of Ruiz-Santana et al was 7.0. The results of the study by Cook et al remained unchanged (table 4) with no difference between sucralfate and placebo (1.26, 0.12 to $12.9, \mathrm{P}=0.70)$. 
Table 2 Patient characteristics, details of interventions, outcomes, and methodological quality score for trials included in our meta-analyses

\begin{tabular}{|c|c|c|c|c|c|c|c|c|}
\hline Trial & Meta-analysis & Setting & $\begin{array}{c}\text { No of } \\
\text { patients }\end{array}$ & $\begin{array}{c}\text { Mean age } \\
\text { (years) }\end{array}$ & Intervention & Control & Outcomes & $\begin{array}{c}\text { Methodological } \\
\text { quality score* }^{*}\end{array}$ \\
\hline $\begin{array}{l}\text { Ruiz-Santana et al, } \\
1991^{11}\end{array}$ & A & ICU & 49 & $\begin{array}{l}R=39 \\
P=39\end{array}$ & $\begin{array}{l}\text { Ranitidine } 50 \mathrm{mg} \text { iv every } 6 \\
\text { hours }\end{array}$ & Placebo & See table 3 & 7 \\
\hline Apte et al, $1992^{12}$ & A & Medical ICU & 34 & $\begin{array}{l}R=27 \dagger \\
P=26 \dagger\end{array}$ & $\begin{array}{l}\text { Ranitidine } 50 \mathrm{mg} \text { iv every } 6 \\
\text { hours }\end{array}$ & Placebo & See table 3 & 6 \\
\hline Metz et al, $1993^{13}$ & A & ICU & $167 \ddagger$ & $\begin{array}{l}R=35.4 \\
P=32.5\end{array}$ & $\begin{array}{l}\text { Ranitidine } 6.25 \mathrm{mg} / \mathrm{hr} \text { by } \\
\text { continuous infusion }\end{array}$ & Placebo & See table 3 & 8 \\
\hline Burgess et al, $1995^{14}$ & A & Surgical ICU & 34 & $\begin{array}{l}R=38.4 \\
P=34.5\end{array}$ & $\begin{array}{l}\text { Ranitidine } 6.25 \mathrm{mg} / \mathrm{hr} \text { by } \\
\text { continuous infusion }\end{array}$ & Placebo & See table 3 & 6 \\
\hline Hanisch et al, $1998^{15}$ & A & Surgical ICU & 114 & $\begin{array}{l}R=55 \\
P=58\end{array}$ & Ranitidine $50 \mathrm{mg}$ tid iv & Placebo & See table 3 & 6 \\
\hline $\begin{array}{l}\text { Ruiz-Santana et al, } \\
1991^{11}\end{array}$ & B & ICU & 54 & $\begin{array}{l}S=37 \\
P=39\end{array}$ & $\begin{array}{l}\text { Sucralfate } 1 \mathrm{~g} \text { by } \\
\text { nasogastric tube every } 4 \\
\text { hours }\end{array}$ & Placebo & See table 4 & 7 \\
\hline Apte et al, $1992^{12}$ & C & Medical ICU & 34 & $\begin{array}{l}\mathrm{R}=27 \dagger \\
\mathrm{P}=26 \dagger\end{array}$ & $\begin{array}{l}\text { Ranitidine } 50 \mathrm{mg} \text { iv every } 6 \\
\text { hours }\end{array}$ & Placebo & See table 5 & 5 \\
\hline Metz et al, $1993^{13}$ & C & ICU & $163 \ddagger$ & $\begin{array}{l}R=35.4 \\
P=32.5\end{array}$ & $\begin{array}{l}\text { Ranitidine } 6.25 \mathrm{mg} / \mathrm{hr} \text { by } \\
\text { continuous infusion }\end{array}$ & Placebo & See table 5 & 7 \\
\hline Hanisch et al, $1998^{15}$ & C & Surgical ICU & 114 & $\begin{array}{l}R=55 \\
P=58\end{array}$ & Ranitidine $50 \mathrm{mg}$ tid iv & Placebo & See table 5 & 6 \\
\hline $\begin{array}{l}\text { Ben-Menachem et al, } \\
1994^{18}\end{array}$ & D & Medical ICU & 200 & $\begin{array}{l}S=60.1 \\
P=59.6\end{array}$ & $\begin{array}{l}\text { Sucralfate } 1 \mathrm{~g} \text { given orally } \\
\text { every } 6 \text { hours }\end{array}$ & Placebo & See table 6 & 7 \\
\hline $\begin{array}{l}\text { Eddleston et al, } \\
1994^{17}\end{array}$ & D & ICU & 26 & $\begin{array}{l}S=47.6 \\
P=54.9\end{array}$ & $\begin{array}{l}\text { Sucralfate } 2 \mathrm{~g} \text { by } \\
\text { nasogastric tube every } 8 \\
\text { hours }\end{array}$ & Placebo & See table 6 & 5 \\
\hline $\begin{array}{l}\text { Pickworth et al, } \\
1993^{19}\end{array}$ & $E$ & Surgical ICU & 83 & $\begin{array}{l}\mathrm{R}=27.3 \\
\mathrm{~S}=26.8\end{array}$ & $\begin{array}{l}\text { Ranitidine } 50 \mathrm{mg} \text { iv every } 6 \\
\text { hours }\end{array}$ & $\begin{array}{l}\text { Sucralfate } 1 \mathrm{~g} \text { by } \\
\text { nasogastric tube } \\
\text { every } 6 \text { hours }\end{array}$ & See table 7 & 6 \\
\hline $\begin{array}{l}\text { Eddleston et al, } \\
1991^{20}\end{array}$ & $E$ & ICU & 60 & $\begin{array}{l}R=54.1 \\
S=44.3\end{array}$ & $\begin{array}{l}\text { Ranitidine } 50 \mathrm{mg} \text { iv every } 6 \\
\text { hours }\end{array}$ & $\begin{array}{l}\text { Sucralfate } 1 \mathrm{~g} \text { by } \\
\text { nasogastric tube } \\
\text { every } 6 \text { hours }\end{array}$ & See table 7 & 7 \\
\hline $\begin{array}{l}\text { Thomason et al, } \\
1966^{25}\end{array}$ & $E$ & $\begin{array}{c}\text { Trauma, } \\
\text { surgical, or } \\
\text { neurosurgical } \\
\text { ICU }\end{array}$ & 160 & $\begin{array}{l}\mathrm{R}=31.0 \dagger \\
\mathrm{S}=27.7 \dagger\end{array}$ & $\begin{array}{l}\text { Ranitidine } 6.25 \mathrm{mg} / \mathrm{hr} \text { by } \\
\text { continuous infusion }\end{array}$ & $\begin{array}{l}\text { Sucralfate } 1 \mathrm{~g} \\
\text { administered } \\
\text { orally then } 1 \mathrm{~g} \text { by } \\
\text { nasogastric tube } \\
\text { every } 6 \text { hours }\end{array}$ & See table 7 & 5 \\
\hline $\begin{array}{l}\text { Prod'hom et al, } \\
1994^{21}\end{array}$ & $E$ & $\begin{array}{l}\text { Medical and } \\
\text { surgical ICU }\end{array}$ & 163 & $\begin{array}{l}R=52.2 \\
S=46.4\end{array}$ & $\begin{array}{l}\text { Ranitidine } 6.25 \mathrm{mg} / \mathrm{hr} \text { by } \\
\text { continuous infusion }\end{array}$ & $\begin{array}{l}\text { Sucralfate } 1 \mathrm{~g} \text { by } \\
\text { nasogastric tube } \\
\text { every } 4 \text { hours }\end{array}$ & See table 7 & 6 \\
\hline O'Keefe et al, $1998^{26} \S$ & $E$ & ICU & 96 & $\begin{array}{l}R=34.3 \\
S=34.2\end{array}$ & $\begin{array}{l}\text { Ranitidine } 0.25 \mathrm{mg} / \mathrm{kg} / \mathrm{hr} \text {, } \\
\text { after loading dose of } 0.5 \\
\mathrm{mg} / \mathrm{kg}\end{array}$ & $\begin{array}{l}\text { Sucralfate } 1 \mathrm{~g} \text { by } \\
\text { nasogastric tube } \\
\text { every } 6 \text { hours }\end{array}$ & See table 7 & 6 \\
\hline Laggner et al, $1989^{22}$ & $E$ & ICU & 32 & $\begin{array}{l}R=60 \\
S=47\end{array}$ & $\begin{array}{l}\text { Ranitidine } 50 \mathrm{mg} \text { iv every } 4 \\
\text { hours }\end{array}$ & $\begin{array}{l}\text { Sucralfate } 1 \mathrm{~g} \text { by } \\
\text { gastric tube every } \\
4 \text { hours }\end{array}$ & See table 7 & 5 \\
\hline Cook et al, $1998^{3}$ & $\mathrm{E}$ & ICU & 1200 & $\begin{array}{l}R=58.8 \\
S=58.7\end{array}$ & $\begin{array}{l}\text { Ranitidine } 50 \mathrm{mg} \text { iv every } 8 \\
\text { hours }\end{array}$ & $\begin{array}{l}\text { Sucralfate } 1 \mathrm{~g} \text { by } \\
\text { nasogastric tube } \\
\text { every } 6 \text { hours }\end{array}$ & See table 7 & 9 \\
\hline Mustafa et al, $1995^{23}$ & $E$ & ICU & 31 & $\begin{array}{l}\text { Not } \\
\text { reported }\end{array}$ & $\begin{array}{l}\text { Ranitidine } 100 \mathrm{mg} \text { iv every } \\
8 \text { hours }\end{array}$ & $\begin{array}{l}\text { Sucralfate } 2 \mathrm{~g} \text { by } \\
\text { nasogastric tube } \\
\text { every } 6 \text { hours }\end{array}$ & See table 7 & 1 \\
\hline
\end{tabular}

${ }^{*}$ In assessment of blinding we assigned score of 1 to single blind studies in which outcome assessor was blind and both caregiver and patient were unblind. †Median.

¥Population studied in effectiveness analysis $(n=167)$ differed from that included in analysis on pneumonia $(n=163)$ because four patients had pneumonia at baseline.

§Results of this clinical trial have in part been reported previously. ${ }^{24}$

$\mathrm{R}=$ ranitidine; $\mathrm{P}=$ placebo; $\mathrm{S}=$ sucralfate; $\mathrm{ICU}=$ intensive care unit.

Incidence of pmeumonia with ranitidine v placebo (metaanalysis C)-Our third meta-analysis included three randomised studies ${ }^{12} 1315$ that compared the incidence of pneumonia between ranitidine and placebo (two of these trials ${ }^{12}{ }^{13}$ had already been included in the metaanalysis by Cook et al). The mean (SD) quality score for these trials was $6.0(1.0)$. The analysis of these three trials (table 5) found no significant difference in the rate of pneumonia with ranitidine and placebo (summary odds ratio $0.98,0.56$ to $1.72, \mathrm{P}=0.94$ for fixed effect model; $1.10,0.45$ to $2.66, \mathrm{P}=0.84$ for random effect model; $\chi^{2}$ for heterogeneity 4.38 , df 2 , $\mathrm{P}=0.11$ ).

Incidence of pneumonia with sucralfate v placebo (metaanalysis D)-Our fourth meta-analysis included two randomised studies ${ }^{17} 18$ that compared the incidence of pneumonia between sucralfate and placebo (both trials had already been included in the meta-analysis by Cook et al). The quality score for these trials was 6.0 (1.4). The analysis of these two trials (table 6) found no significant difference in the rate of pneumonia with sucralfate and placebo (summary odds ratio 2.21, 0.86 to $5.65, \mathrm{P}=0.10$ for fixed effect model; $2.11,0.79$ to 5.64, $\mathrm{P}=0.14$ for random effect model; $\chi^{2}$ for heterogeneity 0.30 , df $1, \mathrm{P}=0.58$ ).

Incidence of pneumonia with ranitidine v sucralfate (meta-analysis E)-Our fifth meta-analysis included eight randomised studies ${ }^{39-26}$ that compared the incidence of pneumonia with ranitidine and sucralfate (five of these trials ${ }^{19-23}$ had already been included in the 
Table 3 Meta-analysis A: rates of gastrointestinal bleeding in patients treated with ranitidine or placebo (five randomised studies)

\begin{tabular}{|c|c|c|c|c|}
\hline \multirow[b]{2}{*}{ Reference } & \multicolumn{2}{|c|}{ Bleeding rate } & \multirow[b]{2}{*}{ Odds ratio $(95 \% \mathrm{CI})$} & \multirow[b]{2}{*}{ Definition of bleeding } \\
\hline & Ranitidine & Placebo & & \\
\hline Ruiz-Santana et al, $1991^{11}$ & $2 / 19$ & $1 / 30$ & 2.81 (0.41 to 28.1$)$ & Acute upper gastrointestinal bleeding \\
\hline Apte et al, $1992^{12}$ & $5 / 16$ & $6 / 18$ & 0.92 (0.23 to 3.65$)$ & Gross gastric bleeding \\
\hline Metz et al, $1993^{13}$ & $0 / 86$ & $1 / 81$ & 0.31 (0.00 to 2.83$)$ & $\begin{array}{l}\text { Bright red blood per nasogastric tube (without including cases with } \\
\text { persistent blood occult positive and "coffee grounds" nasogastric tube } \\
\text { aspirates) }\end{array}$ \\
\hline Burgess et al, $1995^{14}$ & $0 / 16$ & $5 / 18$ & $0.10(0.00$ to 0.86$)$ & $\begin{array}{l}5 \% \text { decrease from baseline in haematocrit occurring at least } 8 \mathrm{~h} \text { after } \\
\text { study drug initiation and haematemesis, haematochezia, bright red } \\
\text { blood per nasogastric tube, or "coffee grounds" nasogastric tube } \\
\text { aspirates }\end{array}$ \\
\hline Hanisch et al, $1998^{15}$ & $3 / 57$ & $2 / 57$ & $1.43(0.29$ to 8.08$)$ & $\begin{array}{l}\text { Bright red blood per gastric tube or melaena combined with } \\
\text { haemodynamic changes (systolic blood pressure }<100 \mathrm{~mm} \mathrm{Hg} \text {, } \\
\text { tachycardia }>100 \text { beats/min) and requirement of blood transfusion (fall } \\
\text { in haemoglobin concentration }>20 \mathrm{~g} / \mathrm{l} \text { within } 24 \text { hours) and endoscopic } \\
\text { identification of bleeding site and activity }\end{array}$ \\
\hline Total & $10 / 194(5 \%)$ & $15 / 204(7 \%)$ & - & \\
\hline
\end{tabular}

Table 4 Meta-analysis B: rates of gastrointestinal bleeding in patients treated with sucralfate or placebo (one randomised study)

\begin{tabular}{|c|c|c|c|c|}
\hline \multirow[b]{2}{*}{ Reference } & \multicolumn{2}{|c|}{ Bleeding rate } & \multirow[b]{2}{*}{ Odds ratio $(95 \% \mathrm{Cl})$} & \multirow[b]{2}{*}{ Definition of bleeding } \\
\hline & Sucralfate & Placebo & & \\
\hline$\overline{\text { Ruiz-Santana et al, } 1991^{11}}$ & $1 / 24(4 \%)$ & $1 / 30(3 \%)$ & $1.26(0.12$ to 12.9$)$ & Acute upper gastrointestinal bleeding \\
\hline
\end{tabular}

Table 5 Meta-analysis C: rates of nosocomial pneumonia in patients treated with ranitidine or placebo (three randomised studies)

\begin{tabular}{|c|c|c|c|c|}
\hline \multirow[b]{2}{*}{ Reference } & \multicolumn{2}{|c|}{ Rate of nosocomial pneumonia } & \multirow{2}{*}{$\begin{array}{l}\text { Odds ratio } \\
(95 \% \mathrm{Cl})\end{array}$} & \multirow[b]{2}{*}{ Definition of pneumonia } \\
\hline & Ranitidine & Placebo & & \\
\hline Apte et al, $1992^{12}$ & $13 / 16$ & $9 / 18$ & $3.86(0.99$ to 19.0$)$ & $\begin{array}{l}\text { Appearance of new infiltrates on chest radiograph or bronchial breath } \\
\text { sounds on examination and positive tracheal culture with fever } \\
\text { axillary temperature }\left(>38^{\circ} \mathrm{C}\right) \text {, leucocytosis }\left(>13000 \mathrm{cell} / \mathrm{mm}^{3}\right) \text {, and } \\
\text { purulent sputum }(>25 \text { leucocytes per low power field) }\end{array}$ \\
\hline Metz et al, $1993^{13}$ & $12 / 84$ & $15 / 79$ & $0.72(0.31$ to 1.61$)$ & $\begin{array}{l}\text { Chest radiograph indicating pulmonary infiltrates and one of six } \\
\text { groupings of clinical findings established by Centers for Disease } \\
\text { Control }\end{array}$ \\
\hline Hanisch et al, $1998^{15}$ & $10 / 57$ & $12 / 57$ & $0.80(0.32$ to 1.99$)$ & $\begin{array}{l}\text { Radiological signs of pneumonia and purulent tracheal secretion or } \\
\text { positive microbiological findings in tracheal aspiration and } \\
\text { temperature }>38^{\circ} \mathrm{C} \text { and leucocytosis }>10000 \mathrm{~mm}^{3}\end{array}$ \\
\hline Total & $35 / 157(22 \%)$ & $36 / 154(23 \%)$ & - & - \\
\hline
\end{tabular}

Table 6 Meta-analysis D: rates of nosocomial pneumonia in patients treated with sucralfate or placebo (two randomised studies)

\begin{tabular}{|c|c|c|c|c|}
\hline \multirow[b]{2}{*}{ Reference } & \multicolumn{2}{|c|}{ Rate of nosocomial pneumonia } & \multirow[b]{2}{*}{ Odds ratio $(95 \% \mathrm{Cl})$} & \multirow[b]{2}{*}{ Definition of pneumonia } \\
\hline & Sucralfate & Placebo & & \\
\hline Ben-Menachem et al, $1994^{18}$ & $12 / 100$ & $6 / 100$ & 2.05 (0.79 to 5.76$)$ & $\begin{array}{l}\text { Each of criteria: chest roentenogram obtained } \geqslant 72 \text { hours after } \\
\text { admission to intensive care that showed new and persistent } \\
\text { infiltrate; fever, leucocytosis, or both; purulent tracheobronchial } \\
\text { secretions; Gram stained sputum showing }>25 \\
\text { polymorphonuclear leucocytes and }<10 \text { squamous epithelial } \\
\text { cells/low power field; recovery of accepted nosocomial } \\
\text { pathogen from sputum culture }\end{array}$ \\
\hline Eddleston et al, $1994^{17}$ & $1 / 14$ & $0 / 12$ & $\begin{array}{c}2.78 \text { (not } \\
\text { computable) }\end{array}$ & $\begin{array}{l}\text { New and progressive infiltrate on chest radiograph; unexplained } \\
\text { reduction in } \mathrm{PaO}_{2} ; \text { positive culture from tracheal aspirate plus } \\
\text { either pyrexia }\left(>38^{\circ} \mathrm{C}\right) \text { or increase in blood leucocyte count } \\
\left(>3 \times 10^{9} \text { cells } / \mathrm{l}\right)\end{array}$ \\
\hline Total & $13 / 114(11 \%)$ & $6 / 112(5 \%)$ & - & \\
\hline
\end{tabular}

meta-analysis by Cook et al). The quality score for these trials was 5.6 (2.3). The analysis of these eight trials (table 7) showed a significantly increased risk of pneumonia with ranitidine compared with sucralfate (summary odds ratio $1.35,1.07$ to $1.70, \mathrm{P}=0.012$ for fixed effect model; $1.51,1.00$ to $2.29, \mathrm{P}=0.05$ for random effect model; $\chi^{2}$ for heterogeneity 12.9 , df $7, \mathrm{P}=0.08$ ).

\section{Discussion}

Our overview of the controlled trials of ranitidine or sucralfate compared with placebo provides a picture of poor effectiveness. The single trial available on sucralfate $^{11}$ does not allow any conclusion to be drawn; the trials on ranitidine $\mathrm{e}^{11-15}$ show no difference compared with placebo.

Our results on ranitidine need to be compared with those previously published by Cook et al. ${ }^{2}$ In their assessment of effectiveness of $\mathrm{H}_{2}$ receptor antagonists Cook et al included five trials that used cimetidine ${ }^{29-33}$ and three trials with negative results that used ranitidine $e^{11-13}$ (plus one trial on ranitidine published in Spanish $^{34}$ and one trial with negative results that used a combination of ranitidine and antacids, ${ }^{35}$ both of which did not meet the criteria for our meta-analysis). Cimetidine is probably effective at statistical levels, as out of the trials that used cimetidine three had positive results, one had significant results in patients at low 
Table 7 Meta-analysis E: rates of nosocomial pneumonia in patients treated with ranitidine or sucralfate (eight randomised studies)

\begin{tabular}{|c|c|c|c|c|}
\hline \multirow[b]{2}{*}{ Reference } & \multicolumn{2}{|c|}{ Pneumonia rate } & \multirow[b]{2}{*}{ Odds ratio $(95 \% \mathrm{CI})$} & \multirow[b]{2}{*}{ Definition of pneumonia } \\
\hline & Ranitidine & Sucralfate & & \\
\hline Pickworth et al, $1993^{19}$ & $5 / 44$ & $6 / 39$ & $0.72(0.21$ to 2.40$)$ & $\begin{array}{l}\text { Presence of new infiltrate in chest } x \text { ray picture and three of: rectal } \\
\text { temperature }>38.5^{\circ} \mathrm{C} \text {, white blood cell count }>10000 \text { cells } / \mathrm{mm}^{3} \text {, } \\
\text { positive sputum culture obtained by leukans trap, or sputum sample } \\
\text { obtained by leukans trap with Gram stain containing many white } \\
\text { blood cells ( }>25 \text { white blood cells, }<10 \text { epithelial cells, and numerous } \\
\text { bacteria/high power field) }\end{array}$ \\
\hline Eddleston et al, $1991^{20}$ & $10 / 30$ & $3 / 30$ & 4.02 (1.18 to 17.1$)$ & $\begin{array}{l}\text { New and progressive infiltrate in chest } x \text { ray picture, unexplained } \\
\text { reduction in } \mathrm{PaO}_{2} \text {, postive culture from tracheal aspirate plus either } \\
\text { pyrexia }\left(>38^{\circ} \mathrm{C}\right) \text { or increase in leucocyte count of }>3000 / \mathrm{mm}^{3}\end{array}$ \\
\hline Thomason et al, $1966^{25}$ & $27 / 80$ & $30 / 80$ & 0.85 (0.45 to 1.61$)$ & $\begin{array}{l}\text { According to Garner et a }{ }^{27} \text { : infiltrate in chest } x \text { ray picture plus three } \\
\text { of: leucocytosis }>10000 \mathrm{cells} / \mathrm{mm}^{3} ; \text { Gram negative organisms on } \\
\text { tracheal or blood culture; tracheal Gram stain showing moderate to } \\
\text { heavy bacteria or polymorphoneutrophils ( }>25 / \text { high power field); } \\
\text { pathogens isolated from tracheal culture; temperature }>38^{\circ} \mathrm{C}\end{array}$ \\
\hline Prod'hom et al, $1994^{21}$ & $21 / 80$ & $10 / 83$ & 2.53 (1.15 to 5.86$)$ & $\begin{array}{l}\text { According to Salata et a }\left.\right|^{28} \text {. new or progressive infiltrate in chest } x \text { ray } \\
\text { picture and at least one of: microbiological or histopathological } \\
\text { evidence of pneumonia; presence of at least two of: leucocytes on } \\
\text { Gram stain, new leucocytosis, temperature increase from }<37.5^{\circ} \mathrm{C} \text { to } \\
>38.5^{\circ} \mathrm{C}\end{array}$ \\
\hline O'Keefe et al, $1998^{26}$ & $14 / 49$ & $10 / 47$ & 1.46 (0.59 to 3.70$)$ & $\begin{array}{l}\text { Leucocytes }>12000 / \mathrm{mm}^{3} \text {, new or changing infiltrate in chest } x \text { ray } \\
\text { picture, temperature }>38.5^{\circ} \mathrm{C} \text { or }<36.5^{\circ} \mathrm{C} \text {, and positive sputum and } \\
\text { Gram stain for specific pathogens. Results of this clinical trial have in } \\
\text { part been reported in separate publication (Maier et al, } 1994^{24} \text { ) }\end{array}$ \\
\hline Laggner et al, $1989^{22}$ & $2 / 16$ & $0 / 16$ & 5.67 (not computable) & $\begin{array}{l}\text { New infilitrate in chest } x \text { ray picture with bronchial colonisation, } \\
\text { leucocytosis }\left(>15000 / \mathrm{mm}^{3}\right) \text {, and fever }>38.5^{\circ} \mathrm{C}\end{array}$ \\
\hline Cook et al, $1998^{3}$ & $114 / 596$ & $98 / 604$ & $1.22(0.91$ to 1.64$)$ & $\begin{array}{l}\text { By consensus of specific pneumonia adjudication committee. Main } \\
\text { criteria included new infiltrate in chest } x \text { ray picture plus at least two } \\
\text { of: temperature }>38.5^{\circ} \mathrm{C} \text { or }<35.0^{\circ} \mathrm{C} \text {; leucocyte count }>10000 \\
\text { cells } / \mathrm{mm}^{3} \text { or }<3000 \mathrm{cell} s / \mathrm{mm}^{3} \text {; purulent sputum or positive culture }\end{array}$ \\
\hline Mustafa et al, $1995^{23}$ & $9 / 16$ & $3 / 15$ & 4.52 (1.12 to 23.6$)$ & No specific definition \\
\hline Total & $202 / 911(22 \%)$ & $160 / 914(18 \%)$ & - & \\
\hline
\end{tabular}

risk, and one had negative results. A separate meta-analysis that we carried out for this purpose (table 8) showed a significant reduction in bleeding (with $\mathrm{P}<0.001$ and $\mathrm{P}<0.01$ according to the fixed effect and the random effect models, respectively).

Can we accept the hypothesis that cimetidine is effective and ranitidine is ineffective? Is this hypothesis pharmacologically plausible? Our separate analyses for individual drugs did not actually settle the entire question. In fact, the cohorts included in the studies that used these two drugs were small and the incidence of events was low, particularly in the case of ranitidine (10 cases of bleeding with the drug $v 15$ without prophylaxis; table 3); so the different results might reflect casual variations in the outcome rather than a true difference between the two $\mathrm{H}_{2}$ receptor antagonists. Another point of uncertainty about cimetidine is the methodological quality of studies published many years ago.

\section{Design of new trials}

Our results indicate that some points of consensus need to be revised. For example, recent randomised studies on prophylaxis for stress ulcers $^{34}$ have invariably compared (unproved) active treatments with one another but no longer use a placebo group, and authoritative recommendations suggest the use of ranitidine for prophylaxis for stress ulcers. ${ }^{36}$

New large scale randomised trials seem to be the only way to resolve this issue. New trials, however, may raise the ethical question of which treatment is appropriate for the control group. One possibility is to give cimetidine to the control group, but this solution raises the question of reusing a drug that has largely been abandoned. Another possibility is to conduct new large scale controlled trials of ranitidine compared with placebo, but the use of placebo can be questionable from an ethical point of view. A third solution could be to design new randomised trials according to a strategy of early treatment of stress ulcer with or without prophylaxis. In this latter case, after randomisation to prophylaxis or placebo the patients could be subjected to intensive gastrointestinal monitoring (for example, by examining nasogastric aspirate at short intervals) and, at the first signs of bleeding, their participation in the trial could be stopped with immediate initiation of an aggressive antisecretory treatment. A drawback of this third solution is that the clinical weight of the end point of early bleeding is less than that of the end point of clinically important bleeding.

\section{Effect on pneumonia}

The results of three meta-analyses that evaluated pneumonia were contradictory in some respects (ranitidine $\mathrm{v}$ placebo and sucralfate $v$ placebo had the same incidence of pneumonia; for ranitidine $v$ sucralfate there was a significantly higher incidence of pneumonia with ranitidine, $\mathrm{P}=0.012$ ). The statistical power of these comparisons was better for meta-analysis $\mathrm{E}$

Table 8 Meta-analysis on effectiveness of cimetidine: rates of gastrointestinal bleeding in patients given cimetidine or placebo

\begin{tabular}{|c|c|c|c|}
\hline \multirow[b]{2}{*}{ Reference } & \multicolumn{2}{|c|}{ Bleeding rate* } & \multirow[b]{2}{*}{ Odds ratio $(95 \% \mathrm{Cl})$} \\
\hline & Cimetidine & Placebo & \\
\hline$\overline{\text { Zinner et } \mathrm{al}^{29}}$ & $14 / 100$ & $20 / 100$ & $0.66(0.31$ to 1.36$)$ \\
\hline Karlstadt et al ${ }^{30}$ & $1 / 54$ & $7 / 33$ & $0.10(0.01$ to 0.43$)$ \\
\hline Groll et a ${ }^{31}$ & $6 / 114$ & $11 / 107$ & 0.50 (0.18 to 1.32$)$ \\
\hline Halloran et a ${ }^{32}$ & $2 / 26$ & $8 / 24$ & 0.20 (0.04 to 0.78$)$ \\
\hline Peura et $\mathrm{a}^{33}$ & $1 / 21$ & $7 / 18$ & 0.11 (0.01 to 0.52$)$ \\
\hline Total & $24 / 315(8 \%)$ & $53 / 282(19 \%)$ & $\dagger$ \\
\hline
\end{tabular}




\section{What is already known on this topic}

Ranitidine and sucralfate are widely used to prevent gastrointestinal bleeding in patients in intensive care

Several recommendations suggest this form of prophylaxis, but both the Food and Drug Administration and European Medicines Evaluation Agency have not given their approval

\section{What this study adds}

This analysis showed that ranitidine and sucralfate do not prevent gastrointestinal bleeding in patients in intensive care

Ranitidine can increase the risk of nosocomial pneumonia under certain circumstances

These findings are based on small numbers of patients and so firm conclusions cannot presently be proposed

Current recommendations on prophylaxis for stress ulcers should be revised according to these results

(1825 patients) than for meta-analysis $\mathrm{C}$ (311 patients); this could in part explain the higher incidence of pneumonia with ranitidine compared with sucralfate but not compared with placebo.

The large trial by Cook et al showed a trend towards an increased incidence of pneumonia with ranitidine $v$ sucralfate (definite pneumonia associated with use of ventilator and pneumonia according to the definition of the Centers for Disease Control and Prevention was more common with ranitidine, at $\mathrm{P}=0.03$ and $\mathrm{P}=0.13$, respectively). ${ }^{3}$ Discrepancies between large trials and meta-analysis have been pointed out in cases where the meta-analysis was conducted before the large trial. ${ }^{37}$ In our study, however, the metaanalysis on pneumonia already included the large trial and, more importantly, its results did not differ much from those of the large trial (odds ratio of $1.35 v 1.18$, respectively).

These arguments related to statistical power and agreement between large scale trials and meta-analyses do not, however, exclude the other explanation that the increase in rates of pneumonia after ranitidine was simply the result of casual variations in the outcome. In this context, the repeated application of statistical testing to many data sets might have contributed to the generation of some significant results.

Our main conclusion is that there are insufficient data on effectiveness to be able to conclude anything one way or the other. This can be an important argument for further trials.

We thank Dr Enrico Tendi, head of the pharmaceutical service of the Careggi hospital, for stimulating the discussion on this topic.

Contributors: AM had the original idea for the present study, set up the project, designed the protocol, organised searches, supervised data extraction, supervised cross checking and validation work, assessed methodological quality of the trials, carried out statistical calculations, and discussed analysis and subsequent results. ST had the original idea for the present study, set up the project, designed the protocol, organised searches, checked accuracy of data extraction, supervised cross checking and validation work, carried out statistical calculations, and discussed analysis and subsequent results. MV was involved in the original project helped to devise protocol, organised searches, extracted data from the studies, assessed methodological quality of the trials, arranged statistical input, collaborated in analyses, and discussed analysis and subsequent results. MG was involved in the original project, helped to devise protocol and organise searches, assessed methodological quality of the trials, collaborated in analyses, and discussed analysis and subsequent results. AC was involved in the original project, helped to devise protocol and organise searches, supervised data extraction, collaborated in analyses, and discussed analysis and subsequent results. The paper was written jointly by $\mathrm{AM}$ and ST. AM is guarantor.

Funding: None.

Competing interests: On other occasions, our group has received support from GlaxoWellcome (Italy) in the following terms: AM has received funds for a member of his staff (MV) working on a pharmacoeconomic project on colon cancer; ST has been reimbursed for attending three symposiums; MV received financial support for the colon cancer project and a fee for consulting about remifentanil; AC has received a fee for speaking and a fee for organising an educational seminar.

\section{Appendix}

Literature search

Our literature search used the PubMed version of Medline on the internet (www.ncbi.nlm.nih.gov/ entrez/). The syntax used for the Medline searches of our five meta-analyses was:

Meta-analysis A: ranitidine AND stress AND eng [LA] AND 1966:2000 [EDAT] AND (Randomized Controlled Trial [PT] OR random* [TW])

Meta-analysis B: sucralfate AND stress AND eng [LA] AND 1966:2000 [EDAT] AND (Randomized Controlled Trial [PT] OR random* [TW])

Meta-analysis C: ranitidine AND pneumonia AND eng [LA] AND 1966:2000 [EDAT] AND Randomized Controlled Trial [PT] OR random* [TW])

Meta-analysis D: sucralfate AND pneumonia AND eng [LA] AND 1966:2000 [EDAT] AND (Randomized Controlled Trial [PT] OR random* [TW])

Meta-analysis E: ranitidine AND sucralfate AND pneumonia AND eng [LA] AND 1966:2000 [EDAT] AND (Randomized Controlled Trial [PT] OR random* [TW])

In this syntax, "eng[LA]" identifies studies published in English language, "1966:2000[EDAT]" selects the year range according to the Entrez date, "Randomized Controlled Trial [PT]" selects this type of trials according to Medical Subjects Headings while "random*"[TW] indicates the word random (in its all variant endings) in the title or abstract; the last two conditions were stated with the "OR" Boolean operator to increase the power of the search.

1 Lam NP, Le PT, Crawford SY, Patel S. National survey of stress ulcer prophylaxis. Crit Care Med 1999;27:98-103.

2 Cook DJ, Reeve BK, Guyatt GH, Heyland DK, Griffith LE, Buckingham L, et al. Stress ulcer prophylaxis in critically ill patients: resolving discordant meta-analyses. JAMA 1996:275:308-14.

3 Cook D, Guyatt G, Marshall J, Leasa D, Fuller H, Hall R, et al. A comparison of sucralfate and ranitidine for the prevention of upper gastrointestinal bleeding in patients requiring mechanical ventilation. Canadian Critical Care Trials Groups. N Engl J Med 1998;338:791-7.

4 Levy MJ, Seelig CB, Robinson NJ, Ranney JE. Comparison of omeprazole and ranitidine for stress ulcer prophylaxis. Dig Dis Sci 1997:49:1255-9

McKibbon KA, Wilczynski NL, Walker-Dilks CJ. How to search and find evidence about therapy. Evidence Based Medicine 1996;1:70.

6 Lau J, Antman EM, Jimenez-Silva J, Kupelnick B, Mosteller F, Chalmers TC. Cumulative meta-analysis of therapeutic trials for myocardial infarction. N Engl J Med 1992;327:248-54.

7 Collins R, Yusuf S, Peto R. Overview of randomised trials of diuretics in pregnancy. BMJ 1985;290:17-23.

8 Der Simonian R, Laird N. Meta-analysis in clinical trials. Cont Clin Trials $1986 ; 7: 177-88$ 
9 Wolf B. On estimating the relation between blood group and disease. Ann Hum Genet 1955;19:251-3.

10 Mannucci PM, Gringeri A, Ammassari M. Antibodies to AIDS and heated factor VIII. Lancet 1985;i:1505-6.

11 Ruiz-Santana S, Ortiz E, Gonzalez B, Bolanos J, Ruiz-Santana AJ, Manzano JL. Stress-induced gastroduodenal lesions and total parenteral nutrition in critically ill patients: frequency, complications, and the value of prophylactic treatment: a prospective, randomized study. Crit Care Med 1991;19:887-91.

12 Apte NM, Karnard DR, Medheakar TP, Tilve GH, Morye S, Bhave GG Gastric colonization and pneumonia in intubated critically ill patient receiving stress ulcer prophylaxis: a randomized controlled trial. Crit Care Med 1992;20:590-3.

13 Metz CA, Livingston DH, Smith JS, Larson GM, Wilson TH. Impact of multiple risk factors and ranitidine prophylaxis on the development of stress-related upper gastrointestinal bleeding: a prospective, multicenter, double-blind, randomized trial. Crit Care Med 1993;21:1844-9.

14 Burgess P, Larson GM, Davidson P, Brown J, Metz CA. Effect of ranitidine on intragastric $\mathrm{pH}$ and stress-related upper gastrointestinal bleeding in patients with severe head injury. Dig Dis Sci 1995;40:645-50.

15 Hanisch EW, Encke A, Naujoks F, Windolf J. A randomized, double-blind trial for stress ulcer prophylaxis shows no evidence of increased pneumonia. Am J Surg 1998;176:453-7.

16 Labattut AG, Santolalla PM, Andres AP. Efficacy of sucralfate in the prevention of upper gastrointestinal stress bleeding in intensive car patients: comparison vs a control group. Clin Intensive Care 1992;3:51925.

17 Eddleston JM, Pearson RC, Holland J, Tooth JA, Vohra A, Doran BH. Prospective endoscopic study of stress erosions and ulcers in critically il adult patients treated with either sucralfate or placebo. Crit Care Med 1994;22:1949-54.

18 Ben-Menachem T, Fogel R, Patel RV, Touchette M, Zarowitz BJ, Hadzijahic $\mathrm{N}$, et al. Prophylaxis for stress-related gastric hemorrhage in the medical intensive care unit: a randomized controlled single blind study. Ann Intern Med 1994;121:568-75.

19 Pickworth KK, Falcone RE, Hoogeboom JE, Santanello SA. Occurrence of nosocomial pneumonia in mechanically ventilated trauma patients: a comparison of sucralfate and ranitidine. Crit Care Med 1993:21:1856-69.

20 Eddleston JM, Vohra A, Scott P, Tooth JA, Pearson RC, McCloy RF, et al. A comparison of the frequency of stress ulceration and secondary pneumonia in sucralfate or ranitidine-treated intensive care unit patients. Crit Care Med 1991;19:1491-6.

21 Prod'hom G, Leuenberger P, Koerfer J, Blum A, Chiolero R, Schaller MD, et al. Nosocomial pneumonia in mechanically ventilated patients receiving antiacid, ranitidine or sucralfate as prophylaxis for stress ulcer. A Intern Med 1994:120:653-69.

22 Laggner AN, Lenz KL, Base W, Druml W, Schneeweiss B, Grimm G. Prevention of upper gastrointestinal bleeding in long term ventilated patients. Sucralfate versus ranitidine. Am J Med 1989;86(6A):81-4
23 Mustafa NA, Akturk G, Ozen I, Koksal I, Erciyes N, Solak M. Acute stres bleeding prophylaxis with sucralfate versus ranitidine and increase of secondary pneumonia in intensive care unit patients. Intensive Care Med 1995;21:287.

24 Maier RV, Mitchell D, Gentilello L. Optimal therapy for stress gastritis. Ann Surg 1994;220:353-60.

25 Thomason MH, Payseur ES, Hakenewerth AM, Norton JH, Mehta B, Reeves R, et al. Nosocomial pneumonia in ventilated trauma patients during stress ulcer prophylaxis with sucralfate, antacid, and ranitidine. $J$ Trauma 1996;41:503-8.

26 O'Keefe GE, Gentilello LM, Maier RV. Incidence of infectious complications associated with the use of histamine 2-receptor antagonists in critically ill trauma patients. Ann Surg 1998;227:120-5.

27 Garner JS, Jarvis WR, Emori TG. CDC definitions for nosocomial infections, 1988. Am J Infect Control 1998;16:128-40.

28 Salata RA, Lederman MM, Schlaes DM, Jacobs MR, Eckstein E, Tweardy $\mathrm{D}$, et al. Diagnosis of nosocomial pneumonia in intubated, intensive care unit patients. Am Rev Respir Dis 1987;135:426-32

29 Zinner MJ, Zuidema GD, Mignosa M, Smith P. The prevention of upper gastrointestinal tract bleeding in patients in an intensive care unit. Surg Gynecol Obstet 1981;153:214-20.

30 Karlstadt RG, Iberti TJ, Silverstein J. Comparison of cimetidine and placebo for the prophylaxis of upper gastrointestinal bleeding due to stress related gastric mucosal damage in the intensive care unit. Intensive Care Med 1990;5:26-32.

31 Groll A, Simon JB, Wigle RD, Taguchi K, Todd RJ, Depew WT. Cimetidine prophylaxis for gastrointestinal bleeding in an intensive care unit. Gu 1986;27:135-40.

32 Halloran LG, Zfass AM, Gayle WE, Wheeler CB, Miller JD. Prevention of acute gastrointestinal complications after severe head injury: a controlled trial of cimetidine prophylaxis. Am J Surg 1980;139:44-8.

33 Peura DA, Johnson LF. Cimetidine for prevention and treatment of gastroduodenal mucosal lesions in patients in an intensive care unit. Ann Intern Med 1985;103:173-7.

34 Brandao A, Alvares-da-Silva MR, Silbemberg J. Ranitidina na profilaxia da emorragia digestiva superior por ulceracoes de stress. Gastrointes Esophageal Dis 1991;10:105-10.

35 Reusser P, Gyr K, Scheidegger D, Buchmann B, Buser M, Zimmerli W. Prospective endoscopic study of stress erosions and ulcers in critically ill neurosurgical patients: current incidence and effect of acid-reducing prophylaxis. Crit Care Med 1990;18:270-4.

36 Tryba M, Cook D. Current guidelines on stress ulcer prophylaxis. Drug 1997;54:581-96.

37 LeLorier J, Gregoire G, Benhaddad A, Lapierre J, Derderian F. Discrepancies between meta-analyses and subsequent large randomized, controlled trials. N Engl J Med 1997;337:536-42.

(Accepted 1 August 2000) 